ACCEPTED MANUSCRIPT

\title{
Enhanced asymmetric transmission in hyperbolic epsilon-near-zero slabs
}

To cite this article before publication: Carlo Rizza et al $2018 \mathrm{~J}$. Opt. in press https://doi.org/10.1088/2040-8986/aacf2f

\section{Manuscript version: Accepted Manuscript}

Accepted Manuscript is "the version of the article accepted for publication including all changes made as a result of the peer review process, and which may also include the addition to the article by IOP Publishing of a header, an article ID, a cover sheet and/or an 'Accepted

Manuscript' watermark, but excluding any other editing, typesetting or other changes made by IOP Publishing and/or its licensors"

This Accepted Manuscript is @ 2018 IOP Publishing Ltd.

During the embargo period (the 12 month period from the publication of the Version of Record of this article), the Accepted Manuscript is fully protected by copyright and cannot be reused or reposted elsewhere.

As the Version of Record of this article is going to be / has been published on a subscription basis, this Accepted Manuscript is available for reuse under a CC BY-NC-ND 3.0 licence after the 12 month embargo period.

After the embargo period, everyone is permitted to use copy and redistribute this article for non-commercial purposes only, provided that they adhere to all the terms of the licence https://creativecommons.org/licences/by-nc-nd/3.0

Although reasonable endeavours have been taken to obtain all necessary permissions from third parties to include their copyrighted content within this article, their full citation and copyright line may not be present in this Accepted Manuscript version. Before using any content from this article, please refer to the Version of Record on IOPscience once published for full citation and copyright details, as permissions will likely be required. All third party content is fully copyright protected, unless specifically stated otherwise in the figure caption in the Version of Record.

View the article online for updates and enhancements. 


\title{
Enhanced asymmetric transmission in hyperbolic epsilon-near-zero slabs
}

Carlo Rizza ${ }^{1, *}$, Xin $\mathbf{L i}^{2}$, Andrea Di Falco ${ }^{2}$, Elia Palange ${ }^{3}$, Andrea Marini ${ }^{4}$, Alessandro Ciattoni ${ }^{1}$

${ }^{1}$ Institute for superconductors, oxides and other innovative materials and devices, National Research Council (CNR-SPIN), Via Vetoio 10, I-67100 L'Aquila, Italy

${ }^{2}$ SUPA, School of Physics and Astronomy, University of St. Andrews, North Haugh, St Andrews, KY169SS, UK

${ }^{3}$ Department of Industrial and Information Engineering and Economics, Via G. Gronchi 18, University of L'Aquila, I-67100 L'Aquila, Italy

${ }^{4}$ Department of Physical and Chemical Sciences, Via Vetoio 10, University of L'Aquila, I-67100 L'Aquila, Italy

E-mail: carlo.rizza@aquila.infn.it

\begin{abstract}
We investigate the asymmetric/transmission for forward and backward propagation of tilted circular polarized optical waves in subwavelength epsilon-nearzero hyperbolic slabs. This chiral-optical effect is solely triggered by anisotropy without resorting to any breaking of reciprocity and chiral symmetries or spatial nonlocal effects. Remarkably, we show that the asymmetric transmission undergoes a dramatic enhancement near the epsilon-near-zero condition. This happens since, close to the zero-crossing point, the extraordinary waves can accumulate the desired propagation phase even though the slab is ultrathin and, by varying excitation angles and slab thickness, we engineer this phase thus achieving a huge asymmetric transmission. The proposed strategy holds promise for realizing ultra-compact and efficient polarization devices in different frequency range even at very high frequency (ultraviolet) since the effect is merely due to anisotropy and it is available without resorting to nanofäbrication processes.
\end{abstract}

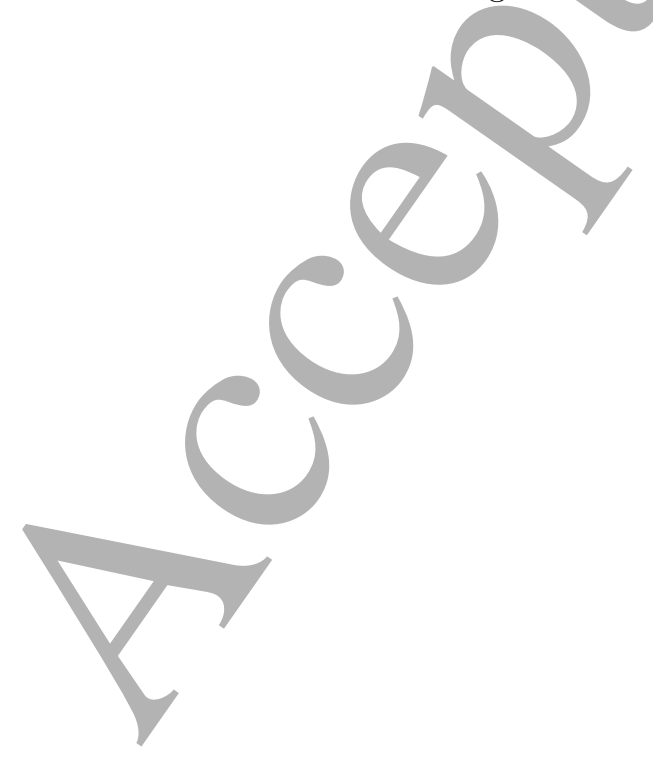




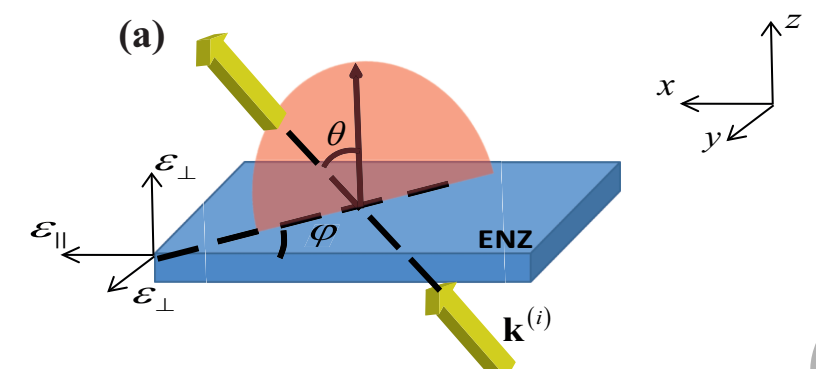

(b) Forward

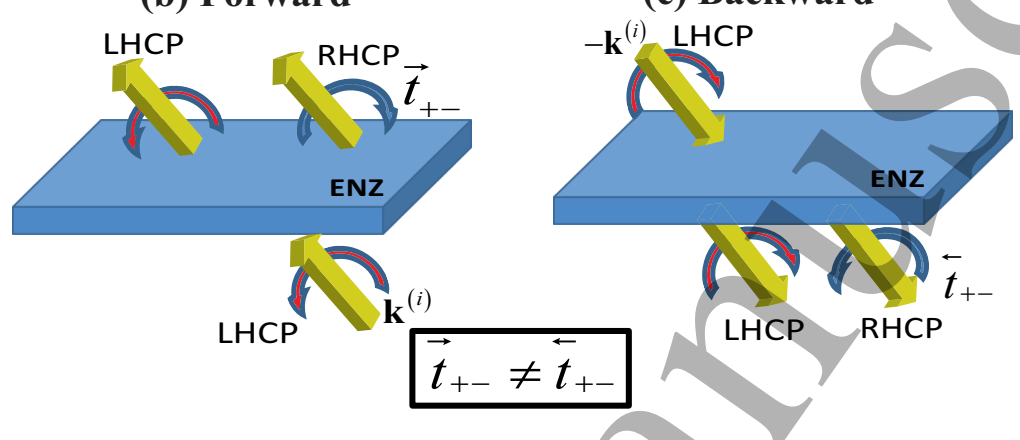

(c) Backward

Figure 1. Geometry of the asymmetric transmission process. (a) Schematic view of the tilted plane wave (yellow arrow) impinging onto an ENZ hyperbolic slab (blue structure) and definition of the coordinate system and angular parameters. Propagation direction is defined as forward for the $\mathbf{k}^{(i)}$ direction (b) and backward for the $-\mathbf{k}^{(i)}$ direction (c). The setup supports circular asymmetric transmission: the forward cross-polarization transmission coefficient is not equal to the backward one. For example, we plot the situation where the incident wave is left-handed circular polarized (LHCP) and the transmitted wave has both the left-handed and the righthanded (RHCP) circular polarized component.

Media with very small permittivity exhibit a number of unique electromagnetic features providing a platform for the radiation manipulation at subwavelenght scales [1]. Epsilon-near-zero (ENZ) condition supports a static-like regime at a given frequency arising from the "stretching" of the wavelength and this key ingredient has an enormous impact for nanophotonics applications shrinking, for example, the device size [3, 4]. Static-like features of the ENZ regime have been exploited to achieve an enormous variety of phenomena such as tunneling of electromagnetic waves through narrow channels [5], slow-light [6,7], and several photonics devices such as highly directive emitters [8], geometry-invariant resonant cavities [9], ultrafast switching devices [10, 11] and ENZ modulators [12, 13].

In the context of nonlinear optics, ENZ media have attracted a great deal of interest since ENZ regime is able to trigger several non-linearity enhancement mechanisms. For example, the ubiquitous phase-matching, essential to achieve strong nonlinear coupling, is automatically satisfied in zero-index media [14]. Furthermore, one can achieve high field intensity in a nonlinear ENZ medium due to the extreme effects appearing at the boundary where the normal electric field component undergoes a drammatic enhancement $[15,16,17,18,19]$. In Ref.[20], authors theoretically suggested that ENZ 
media enable the observation of extreme nonlinear effects since the nonlinear polarization is comparable with or even greater than the linear part of the overall dielectric response and, recently, such extreme nonlinear regime has been investigated in different setups $[21,22,23,24,25,26,27,28]$.

The strategy to reduce the predominant local contribution has also been exploited to emphasize the non-reciprocal and nonlocal responses [29, 30, 31]. Pollard et al. have observed strong non-localities in ENZ metamaterials even supporting additional transverse magnetic waves [32]. Recently, Rizza et al. [33] have shown that the electromagnetic response of a chiral ENZ metamaterial is strongly affected by the firstorder spatial dispersion.

An additional consequence of the ENZ static-like regime is the boosting of transverse magnetic (TM) and transverse electric (TE) asymmetric response in ultrathin ENZ slab. This effect has been demonstrated to support vortex generation [4], efficient polarization conversion in ultrathin waveplates [2] and enhancement of the light spinHall effect [34].

In this paper, we show that an ultrathin ENZ homogeneous slab can support a huge asymmetric transmission for forward and backward propagation of tilted circular polarized optical waves. Generally, asymmetric transmission and the associated diodelike response can be achieved by breaking either the Lorentz reciprocity or the spatial inversion symmetry. Reciprocity is broken in magneto-optical materials [35, 36], in nonlinear media [37], or in materials where the refractive index is spatio-temporal modulated [38], whereas spatial symmetry breaking is hosted by chiral materials and it has been investigated in several configurations [39, 40, 41, 42].

In addition, Plum et al. [43] demonstrated that asymmetric transmission can occur in achiral metamaterial structures (not characterized either by the 2D or 3D chiral asymmetry). The effect ensues from the mutual orientation of the structure and the incident light propagation direction (pseudo/extrinsic chirality) and it is supported by any planar lossy periodic array containing particles of any symmetry. In this context, it is worth noting that extrinsic chirality arises by different fundamental mechanisms. In a 1D metamaterial, the structure can exhibit one-dimensional chirality (i.e., its mirror image cannot be superposed onto it by using translations without resorting to rotations) [33]. In higher symmetry structures, such as a hole array on a square lattice in a metallic film, strong chiroptical effects are due to second-order spatial dispersion and hence they can not be explained in the framework of a local effecive medium theory [45]. In addition, extrinsic chirality appears if the considered experimental setup possesses a definite handedness and it can be described by three noncoplanar unit vectors. For example, nonlinear chiral effects can be observed by impinging a tilted circularlypolarized wave onto a homogeneous film with an in-plane anisotropy axis [44]. Here, exploiting the asymmetric chiral response offered by an anisotropic slab, we investigate the asymmetric transmission for forward and backward propagation of tilted circular polarized optical waves. We prove that the diode-like response is dramatically enhanced if the slab is hyperbolic in the ENZ condition. This enhancement arises from the exotic 


\section{Enhanced asymmetric transmission in hyperbolic epsilon-near-zero slabs}

feature of ENZ hyperbolic slabs of subwavelength thickness which can even exhibit etalon resonances [46].

We consider a slab of thickness $L$ illuminated by a monochromatic plane wave of wavelength $\lambda$ whose electric field is $\operatorname{Re}\left[\mathbf{E}(z) \exp \left(-i 2 \pi c t / \lambda+i k_{y} y+i k_{x} x\right)\right]$ and impinging from vacuum with incident angles $\theta$ and $\varphi$, as reported in Fig.1(a), so that its wave vector is $\mathbf{k}^{(i)}=\mathbf{k}_{\perp}+k_{z}^{(i)} \hat{\mathbf{e}}_{z}=k_{0}\left(\sin \theta \cos \varphi \hat{\mathbf{e}}_{x}+\sin \theta \sin \varphi \hat{\mathbf{e}}_{y}+\cos \theta \hat{\mathbf{e}}_{z}\right)\left(k_{0}=2 \pi / \lambda\right.$, $\left.\mathbf{k}_{\perp}=k_{x} \hat{\mathbf{e}}_{x}+k_{y} \hat{\mathbf{e}}_{y}\right)$. The considered slab is a homogeneous uniaxially anisotropic medium (a natural homogeneous material or a metamaterial), where the optical axis is oriented along the $x$-axis so that the electromagnetic response is described by the permittivity tensor $\varepsilon=\operatorname{diag}\left(\varepsilon_{\|}, \varepsilon_{\perp}, \varepsilon_{\perp}\right)$. Imposing the transverse momentum conservation across the slab interfaces for a given transversal wave vector $\mathbf{k}_{\perp}$, there are two forward waves excited within the slab, i.e., the ordinary and extraordinary plane waves, whose their longitudinal wave vectors are

$$
\begin{aligned}
& k_{z}^{(o)}=\sqrt{k_{0}^{2} \varepsilon_{\perp}-k_{\perp}^{2}}, \\
& k_{z}^{(e)}=\sqrt{k_{0}^{2} \varepsilon_{\|}-\left(k_{x}^{2} \frac{\varepsilon_{\|}}{\varepsilon_{\perp}}+k_{y}^{2}\right)} .
\end{aligned}
$$

It is worth noting that the longitudinal wave vector of the extraordinary plane wave shows a singularity for $\epsilon_{\perp}=0$ with $k_{x} \neq 0, \epsilon_{\|} \neq 0$, so that the extraordinary waves can accumulate the desired propagation phase even though the slab has a subwavelength thickness and/we obtain an enormous difference between the dynamics of the extraordinary and the ordinary waves inside the considered medium. In order to demonstrate the asymmetric transmission of circularly polarized waves as described in Fig.1 (b) and (c), we consider at first the the forward scattering in the TM (p) and TE (s) linear polarization basis $\hat{\mathbf{e}}_{p}=\cos \theta \cos \varphi \hat{\mathbf{e}}_{x}+\cos \theta \sin \varphi \hat{\mathbf{e}}_{y}-\sin \theta \hat{\mathbf{e}}_{z}$, $\hat{\mathbf{e}}_{s}=-\sin \varphi \hat{\mathbf{e}}_{x}+\cos \varphi \hat{\mathbf{e}}_{y}$. After matching the fields at the slab interfaces, we obtain the forward transmission matrix, $\overrightarrow{\mathbf{T}}_{l}$, connecting the components of the incident $\mathbf{E}^{(i)}$ and the transmitted $\mathbf{E}^{(t)}$ electric fields, namely

$$
\left(\begin{array}{l}
E_{p}^{(t)} \\
E_{s}^{(t)}
\end{array}\right)=\left(\begin{array}{ll}
\vec{t}_{p p} & \vec{t}_{p s} \\
\vec{t}_{s p} & \vec{t}_{s s}
\end{array}\right)\left(\begin{array}{l}
E_{p}^{(i)} \\
E_{s}^{(i)}
\end{array}\right)=\overrightarrow{\mathbf{T}}_{l}\left(\begin{array}{c}
E_{p}^{(i)} \\
E_{s}^{(i)}
\end{array}\right) .
$$

Note that, for the considered slab, anisotropy yields to polarization conversion where the cross-polarization transmission coefficient are identical and non-vanishing, viz. $\vec{t}_{p s}=$ $\vec{t}_{s p} \neq 0$ [47]. On the other hand, in the circular polarization basis $\hat{\mathbf{e}}_{+}=1 / \sqrt{2}\left(\hat{\mathbf{e}}_{p}+i \hat{\mathbf{e}}_{s}\right)$, $\hat{\mathbf{e}}_{-}=1 / \sqrt{2}\left(\hat{\mathbf{e}}_{p}-i \hat{\mathbf{e}}_{s}\right)$, the forward transmission matrix

$$
\overrightarrow{\mathbf{T}}_{c}=\left(\begin{array}{cc}
\vec{t}_{++} & \vec{t}_{+-} \\
\vec{t}_{-+} & \vec{t}_{--}
\end{array}\right)
$$


Enhanced asymmetric transmission in hyperbolic epsilon-near-zero slabs has the entries

$$
\begin{aligned}
& \vec{t}_{++}=\frac{1}{2}\left(\vec{t}_{p p}+\vec{t}_{s s}\right), \\
& \vec{t}_{+-}=\frac{1}{2}\left(\vec{t}_{p p}-\vec{t}_{s s}-i 2 \vec{t}_{p s}\right), \\
& \vec{t}_{-+}=\frac{1}{2}\left(\vec{t}_{p p}-\vec{t}_{s s}+i 2 \vec{t}_{p s}\right), \\
& \vec{t}_{--}=\frac{1}{2}\left(\vec{t}_{p p}+\vec{t}_{s s}\right),
\end{aligned}
$$

Now, we consider the backward excitation as depicted in Fig.1(c) where the backward incident wave propagates along $-\mathbf{k}^{(i)}$. According to the reciprocal theorem [48], asymmetric transmissions in the above linearly and circularly polarized bases are usually characterized by the parameters

$$
\begin{aligned}
& \Delta_{l}=\left|\vec{t}_{s p}\right|^{2}-\left|\overleftarrow{t}_{s p}\right|^{2}=\left|\vec{t}_{s p}\right|^{2}-\left|\vec{t}_{p s}\right|^{2}, \\
& \Delta_{c}=\left|\vec{t}_{+-}\right|^{2}-\left|\overleftarrow{t}_{+-}\right|^{2}=\left|\vec{t}_{+-}\right|^{2}-\left|\vec{t}_{-+}\right|^{2}
\end{aligned}
$$

Using the above expressions for entries of the transmission matrices in the linear basis we obtain $\Delta_{l}=0$, i.e. asymmetric transmission does not occur for linearly polarized waves. Conversely, using Eqs.(4), we get

$$
\Delta_{c}=2|\Delta t|\left|\vec{t}_{s p}\right| \sin (\Delta \psi)
$$

where we define $\Delta t=\vec{t}_{p p}-\vec{t}_{s s}$ and $\Delta \psi=\operatorname{Arg}\left(\Delta t \vec{t}_{p s}^{*}\right)$. From Eq.(7), it is evident that the asymmetric transmission for tilted plane waves is a consequence of both the transverse electric and transverse magnetic polarization asymmetric response $\Delta t \neq 0$ and the presence of the linear cross-polarization conversion $\vec{t}_{s p} \neq 0$, two ingredients naturally offered by an uni-axial medium. As stated above, in the ENZ regime, the extraordinary waves can accumulate the desired propagation phase so that one can engineer this phase and, in turn, the values of $\Delta t$ and $\vec{t}_{s p}$ for achieving a significant enhancement of the asymmetric transmission.

To demonstrate the enhancement of the asymmetric transmission in ENZ regime, we consider a slab made of monocrystalline bismuth which is a homogeneous naturallyoccurring material showing a hyperbolic response for far-infrared frequencies. In this frequency range, the electromagnetic response of bismuth can be described by the Drude model,

$$
\begin{aligned}
& \varepsilon_{\perp}=\epsilon_{\infty_{\perp}}\left(1-\frac{\omega_{\perp}^{2}}{\omega^{2}+i \omega \tau^{-1}}\right), \\
& \varepsilon_{\|}=\epsilon_{\infty_{\|}}\left(1-\frac{\omega_{\|}^{2}}{\omega^{2}+i \omega \tau^{-1}}\right),
\end{aligned}
$$

where $\omega=2 \pi c / \lambda$ is the angular frequency, $\epsilon_{\infty_{\perp}}=76, \epsilon_{\infty_{\|}}=110, \tau=0.1 \mathrm{~ns}$ is the relaxation time, $\omega_{\perp}=186 \mathrm{~cm}^{-1}$ and $\omega_{\|}=158 \mathrm{~cm}^{-1}$ are the plasma frequencies [49]. To discuss the role of ENZ condition, we neglect the material loss and we fix the angle $\theta=60 \mathrm{deg}$ and $\lambda=53.78 \mu \mathrm{m}$ (where $\varepsilon_{\|}=30.6$ and $\varepsilon_{\perp}=-0.05$ ). In Fig.2(a), 
Enhanced asymmetric transmission in hyperbolic epsilon-near-zero slabs

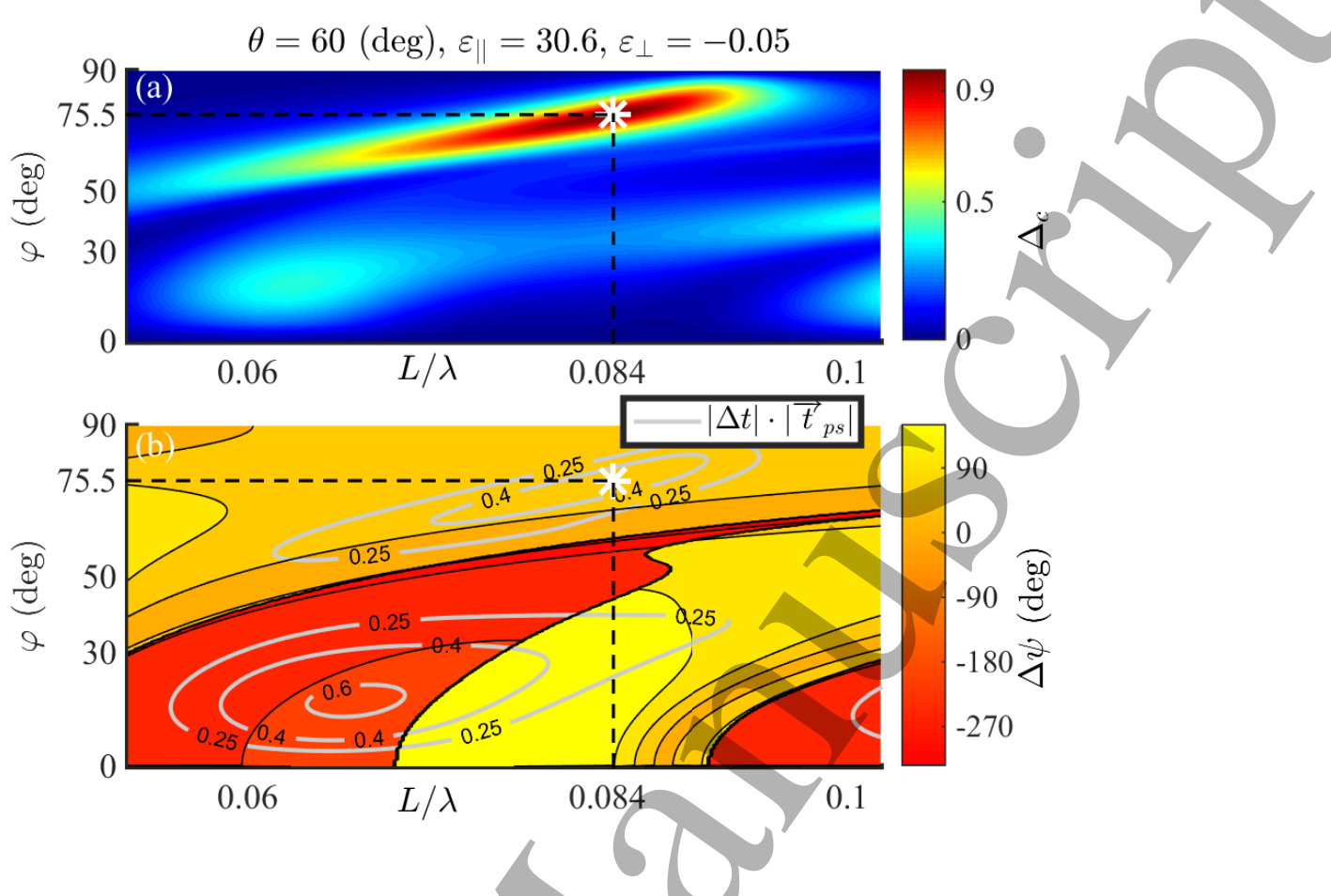

Figure 2. (a) Asymmetric transmission $\Delta_{c}$ as a function of $\varphi$ and the normalized slab thickness $L / \lambda$. (b) $\Delta \psi=\operatorname{Arg}\left(\Delta t \vec{t}_{p s}^{*}\right)$ as a function of $\varphi$ and the normalized slab thickness $L / \lambda$. We obtain a near-unity asymmetric transmission for $|\Delta t|\left|\vec{t}_{p s}\right| \simeq 0.5$ and $\Delta \psi \simeq 90 \mathrm{deg}$ (marked with white-stars). We contourplot the function $|\Delta t|\left|\vec{t}_{p s}\right|$ with grey solid lines.

we plot the circular asymmetric transmission $\Delta_{c}$ as a function of the incident angle $\varphi$ and the normalized slab thickness $L / \lambda$. It is evident that the phenomenon of the asymmetric transmission is relevant for the considered configuration and it becomes maximum (viz. $\Delta_{c}=0.97$ with $\left|\vec{t}_{R L}\right|^{2}=0.022$ and $\left|\vec{t}_{L R}\right|^{2}=0.996$ ) at $\varphi=75.5 \mathrm{deg}$ and at $L=0.084 \lambda \simeq 4.5 \mu \mathrm{m}$ (the white star in Fig.2(a)). As stated above, the effect is triggered from the transverse electric and transverse magnetic polarization asymmetric response and from the polarization conversion. In Fig.2(b), we highlight that these two phenomena are efficient and we obtain a near-unity asymmetric transmission for $|\Delta t|\left|\vec{t}_{p s}\right| \simeq 0.5$ (see grey contour lines) and $\Delta \psi \simeq 90 \mathrm{deg}$ (marked with the white star in Fig.2(b)). In the ENZ regime where $-1 \ll \varepsilon_{\perp}<0$ and $\varepsilon_{\|}>0$ and $k_{\perp} \neq 0$, we have that the ordinary wave is evanescent $\left(k_{z}^{(o)}=\sqrt{-k_{\perp}^{2}}\right)$, whereas the extraordinary wave is a propagating mode and it can accumulate the desired propagation phase even if the slab is ultrathin.

In fact, close to the ENZ condition, hyperbolic dispersion can support extraordinary waves whose longitudinal wave vectors are suitable large $\left(k_{z}^{(e)} \gg k_{0}\right)$, thus activating even etalon resonances and enabling a huge diode-like response of the system. In summary, the effect is supported and triggered by the slab anisotropic response and it is dramatically enhanced in the ENZ hyperbolic regime. In Fig.3, we plot $\left|\Delta_{c}\right|$ as a function of $\varphi$ and $\theta$ setting $\lambda=53.78 \mu \mathrm{m}, L=0.084 \lambda$ and for the same slab parameters 
Enhanced asymmetric transmission in hyperbolic epsilon-near-zero slabs

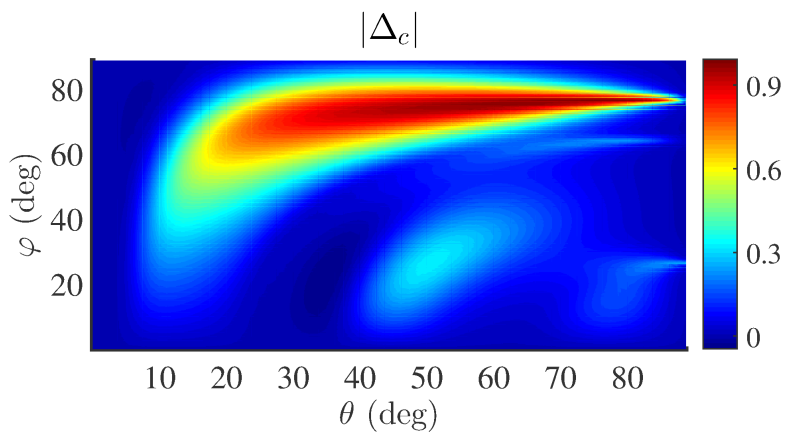

Figure 3. The absolute value of $\Delta_{c}$ as a function of $\varphi$ and $\theta$ setting $\lambda=53.78 \mu \mathrm{m}$, $L=0.084 \lambda, \varepsilon_{\|}=30.6$ and $\varepsilon_{\perp}=-0.05$.

(a)
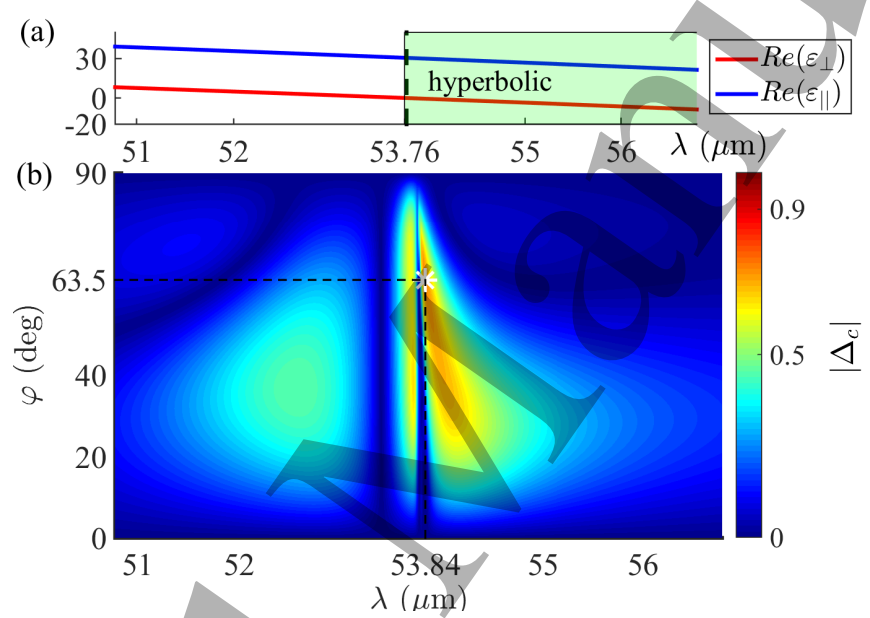

Figure 4. (a) Real part of the bismuth dielectric constants $\varepsilon_{\perp}, \varepsilon_{\|}$reported in Eqs.(8) (the green area indicates hyperbolic region). The absolute value of $\Delta_{c}$ as a function of $\varphi$ and $\lambda$ for a bismuth slab with $L=4.5 \mu \mathrm{m}$ and $\theta=60 \mathrm{deg} .\left|\Delta_{c}\right|$ attains its maximum $\left(\Delta_{c}=0.73\right)$ for $\varphi=63.5(\mathrm{deg})$ and $\lambda=53.84 \mu \mathrm{m}$ (marked with a white star).

of Fig.2. It is interesting to note that the asymmetric transmission can be very high for $\theta>45(\mathrm{deg})$, whereas the effect fully disappears for small angles $(\theta<10(\mathrm{deg}))$.

As expected, the imaginary part of $\varepsilon_{\perp}$, associated to the slab losses, plays a relevant role in the ENZ regime. In Fig.4, we show the circular asymmetric transmission $\left|\Delta_{c}\right|$ as a function of $\lambda$ and $\varphi$ for a bismuth slab, whose the dielectric response is given by Eqs.(8) and setting $L=4.5 \mu \mathrm{m}$ and $\theta=60 \mathrm{deg}$. The absolute value of $\Delta_{c}$ attains its maximum $\left(\Delta_{c} \simeq 0.73\right.$ with $\left|\vec{t}_{R L}\right|^{2}=0.786$ and $\left.\left|\vec{t}_{L R}\right|^{2}=0.057\right)$ for $\varphi=63.5(\mathrm{deg})$ and $\lambda=53.84$ $\mu \mathrm{m}$, where the slab presents an ENZ hyperbolic response (i.e., $\varepsilon_{\|}=30.40+i 0.02$ and $\left.\varepsilon_{\perp}=-0.22+i 0.02\right)$. The considered setup shows significant advantages such as compact size, no need of complex time-consuming fabrication processes and high circular asymmetric transmission without resorting to nonlinearity and external magnetic fields. Clearly, losses are not negligible in the ENZ regime, even if $\operatorname{Im}\left(\varepsilon_{\perp}\right) \ll \operatorname{Re}\left(\varepsilon_{\perp}\right)$, so that their presence is detrimental and, in this case, we do not attain the ideal near-unity asymmetric transmission. In addition, losses depends on the purity of the sample, so 


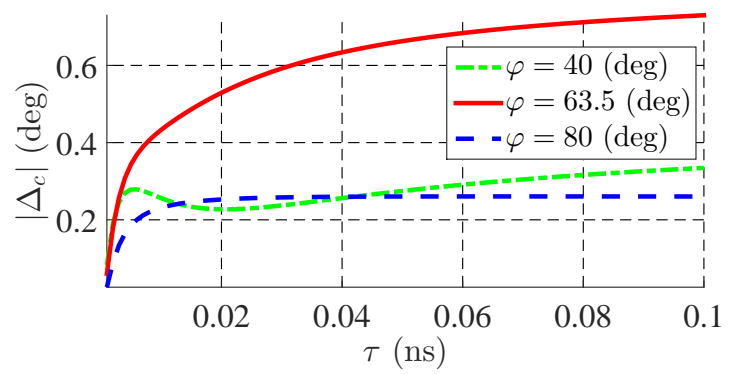

Figure 5. $\Delta_{c}$ as a function of $\tau$ for $\varphi=40$ (deg), $\varphi=63.5$ (deg), $\varphi=80$ (deg) setting $\lambda=53.84 \mu \mathrm{m}, \theta=60 \mathrm{deg}$ and $L=4.5 \mu \mathrm{m}$.

that, for completeness, in Fig.5 we plot the absolute value of $\Delta_{c}$ as a function of the relaxation time $\tau$ (defined in Eqs.(8)) for different values of $\varphi$, setting $\lambda=53.84 \mu \mathrm{m}$, $\theta=60 \mathrm{deg}$ and $L=4.5 \mu \mathrm{m}$. As stated above, the asymmetric transmission attains a maximum for $\tau=0.1 \mathrm{~ns}$ whereas the asymmetry decreases for smaller values of the relaxation time. Therefore, we conclude that the effect is modified by losses but, surprisingly, it persists in the situation where the slab is ultrathin.

In the considered example, we have discussed the asymmetric transmission occurring in a natural ENZ hyperbolic material. Unfortunately, they are not available in some spectral region (e.g. in the optical range) [50], and thus one has to consider a metamaterial structure (whose response can be tuned by changing both its geometry and inclusion materials) for achieving the effect at the desired frequency. In particular, we focus our attention on a subwavelength binary grating, made of silver and air whose filling fraction are $f_{A g}=0.5, f_{\text {Air }}=0.5$, respectively, and whose stacking direction is along the $x$-axis. The silver dielectric permittivity is described by the Drude model $\varepsilon_{A g}=\epsilon_{b}-\omega_{p}^{2} /\left(\omega^{2}+i \alpha \omega\right)$ (where $\epsilon_{b}=5, \omega_{p}=14 \cdot 10^{15} \mathrm{~Hz}$ and $\alpha=0.32 \cdot 10^{15} \mathrm{~Hz}$ ). Note that we increase the parameter $\alpha$ by a factor of 10 to consider the layer size effect [51]. In the homogenized regime (namely, in the situation where $\Lambda \ll \lambda$ ), the effective electromagnetic response is described by the permittivity tensor $\operatorname{diag}\left(\varepsilon_{\|}, \varepsilon_{\perp}, \varepsilon_{\perp}\right)$, where $\varepsilon_{\perp}=f_{A g} \varepsilon_{A g}+f_{A i r}$ and $\varepsilon_{\|}=\left(f_{A g} / \varepsilon_{A g}+1 / f_{A i r}\right)^{-1}$, so that the metamaterial response is characterized by the ENZ crossing point $\operatorname{Re}\left(\varepsilon_{\perp}\right)=0$ at $\lambda=0.4 \mu \mathrm{m}$ as reported in Fig.6(a). In Fig.6(b), we plot the $\Delta_{c}$ parameter evaluated for $\theta=60 \mathrm{deg}$ and $L=30$ $\mathrm{nm}$. The asymmetric transmission attains its maximum $\Delta_{c} \simeq 0.2$ at $\lambda=0.42 \mu \mathrm{m}$ and $\varphi=45$ deg where $\varepsilon_{\|}=1.32+i 0.01$ and $\varepsilon_{\perp}=-0.14+i 0.14$.

In order to verify the results of the effective medium theory (EMT) and to discuss the impact of spatial nonlocality on the considered asymmetric transmission, we consider the numerical implementation of the rigorous coupled-wave analysis technique [52] for obtaining the 0th-order transmission coefficients and, hence, for evaluating the asymmetric transmission $\Delta_{c}$. In the Fig.6(c), for $\varphi=45 \mathrm{deg}$ and $\theta=60 \mathrm{deg}$, we report the comparison between the asymmetric transmission evaluated with EMT (black solid line) and those predicted by the rigorous coupled-wave analysis for different values of the spatial period $\Lambda$. Clearly, close to the ENZ crossing point, non-locality affected 
Enhanced asymmetric transmission in hyperbolic epsilon-near-zero slabs

(a)
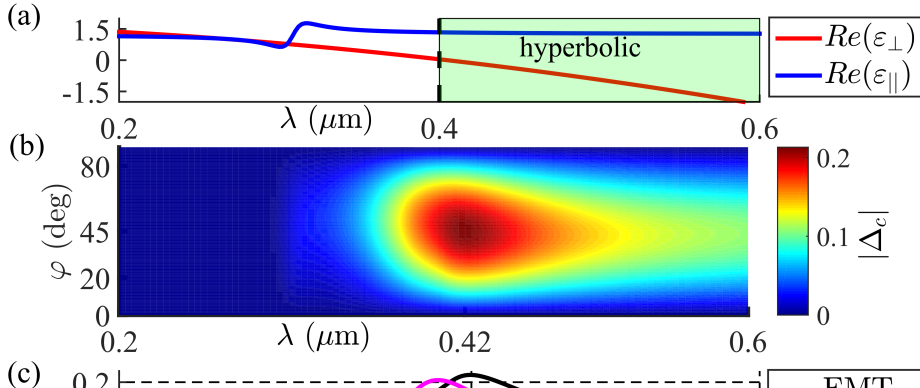

(c)

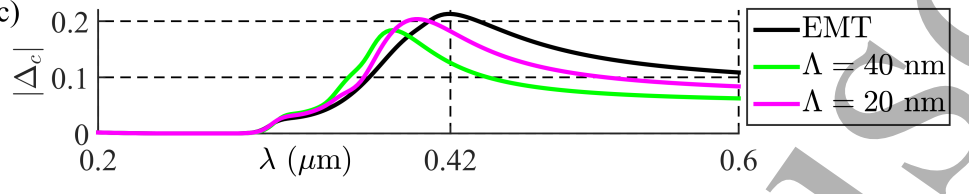

Figure 6. (a) Real part of the metamaterial effective permittivity as a function of $\lambda$ (the shadow area indicates hyperbolic region). (b) $\Delta_{c}$ parameter as a function of $\varphi$ and $\lambda$ for $\theta=60 \mathrm{deg}$ and $L=30 \mathrm{~nm}$. (c) Comparison between the asymmetric transmission evaluated with EMT (black solid line) and those predicted by the rigorous coupled-wave analysis for different values of the spatial period $\Lambda$.

the results [33] and, here, it slightly reduces the value of the $\Delta_{c}$ parameter. As a consequence, the results of the rigorous coupled-wave analysis technique assures that the asymmetric transmission is purely related to the structure homogeneous response.

In conclusion, we demonstrate that an ultrathin ENZ hyperbolic slab supports a nearly $100 \%$ asymmetric transmission for circularly polarized tilted waves. The optical like-diode response is merely due to slab anisotropic response and it depends on neither non-reciprocity nor chirality nor spatial nonlocality so that the effect is available in natural hyperbolic materials. We believe that our investigation constitutes a fundamental step for the realization of compact polarization devices at different frequency ranges.

\section{Acknowledgement}

CR and AC thank the U.S. Army International Technology Center Atlantic for financial support (Grant No. W911NF-14-1-0315). This work has partially been supported by the CNR-SPIN Seed Project No. B52F17001370005. AM acknowledges support from the "Rita Levi Montalcini" programme for the recruitment of young researchers. CR and EP acknowledges support from the project NANOPREPAINT - PAR FSC Abruzzo 2007-2013 - Linea di Azione I.1.1.a.

[1] Liberal I and Engheta N 2017 Nature Photonics 11149.

[2] Ginzburg P Rodrguez Fortuno F J Wurtz G A Dickson W Murphy A Morgan F Pollard R J Iorsh I Atrashchenko A Belov P A Kivshar Y S Nevet A Ankonina G Orenstein M and Zayats A V 2013 Opt. Express 2114907.

[3] Zhong S Ma Y and He S 2014 Appl. Phys. Lett. 105, 023504.

[4] Ciattoni A Marini A and Rizza C 2017 Phys. Rev. Lett. 118104301.

[5] Silveirinha M and Engheta N 2006 Phys. Rev. Lett. 97157403.

[6] Ciattoni A, Marini A Rizza C Scalora M and Biancalana F 2013 Phys. Rev. A 87053853. 
Enhanced asymmetric transmission in hyperbolic epsilon-near-zero slabs

[7] Newman W D Cortes C L Atkinson J Pramanik S DeCorby R G and Jacob Z 2015 ACS Photonics 2,2 .

[8] Alu A Silveirinha M Salandrino A and Engheta N 2007 Phys. Rev. B 75155410.

[9] Liberal I Mahmoud A M and Engheta N 2016 Nat. Commun. 710989.

[10] Kinsey N DeVault C Kim J Ferrera M Shalaev V M and Boltasseva A 2015 Optica 2616.

[11] Yang Y Kelley K Sachet E Campione S Luk T S Maria J Sinclair M B and Brener I 2017 Nat. Photonics 11390.

[12] Lee H W Papadakis G Burgos S P Chander K Kriesch A Pala R Peschel U and Atwater H A 2014 Nano Lett. 146463.

[13] Wood M G Campione S Parameswaran S Luk T S Wendt J R Serkland D K and Keeler G A 2018 Optica 5233.

[14] Suchowski H OBrien K Wong Z J Salandrino A Yin X and Zhang X 2013 Science 342, 1223.

[15] Campione S De Ceglia D Vincenti M A Scalora M and Capolino F 2013 Phys. Rev. B 87035120.

[16] Vincenti M A De Ceglia D Ciattoni A and Scalora M 2011 Phys. Rev. A 84063826.

[17] Ciattoni A and Spinozzi E 2012 Phys. Rew. A 85043806.

[18] Capretti A Wang Y Engheta N and Dal Negro L 2015 Opt. Lett. 401500.

[19] Luk T S De Ceglia D Liu S Keeler G A Prasankumar R P Vincenti M A Scalora M Sinclair M B and Campione S 2015 Appl. Phys. Lett. 106151103.

[20] Ciattoni A Rizza C and Palange E 2010 Phys. Rev. A 81043839.

[21] Marini A and Garcia De Abajo F J 2016 Sci.Rep. 6, 20088.

[22] Ciattoni A Rizza C Marini A Di Falco A Faccio D and Scalora M 2016 Laser Photonics Rev. 10 517.

[23] Ciattoni A Marini A and Rizza C 2016 Optics Letters 413102.

[24] Alam M Z De Leon I and Boy R W 2016 Science 352795.

[25] Kaipurath R M Pietrzyk M Caspani L Roger T Clerici M Rizza C Ciattoni A Di Falco A and Faccio D 2016 Sci. Rep. 6, 27700.

[26] Caspani L Kaipurath R P M Clerici M Ferrera M Roger T Kim J Kinsey N Pietrzyk M Di Falco A Shalaev V M Boltasseva A and Faccio D, 2016 Phys. Rev. Lett. 2016116233901.

[27] Prain A Vezzoli S Westerberg N Roger T and Faccio D 2017 Phys. Rev. Lett. 118133904.

[28] Alam M Z Schultz S A Upham J De Leon I and Boyd R W 2018 Nat. Photonics 1279.

[29] Tretyakov S Nefedov I Sihvola A Maslovski S and Simovski C 2003 J. Electromagn. Waves Appl. 17695.

[30] Davoyan A R Mahmoud A M and Engheta N 2013 Opt. Express 213279.

[31] De Ceglia D Scalora M Vincenti M A Campione S Kelleye K Runnerstrome E L Maria J Keeler G A and Luk T S 2017 ArXiv:1712.03169.

[32] Pollard R J Murphy A Hendren W R Evans P R Atkinson R Wurtz G A Zayats A V and Podolskiy V A 2009 Phys. Rev. Lett. 102127405.

[33] Rizza C Di Falco A Scalora M and Ciattoni A 2015 Phys. Rev. Lett. 115057401.

[34] Zhu W and She W 2015 Opt. Lett. 402961.

[35] Bi L Jiang J P Kim D H Dionne G F Kimerling L C and Ross C A 2011 Nature Photonics 5758.

[36] Wang Z Chong Y Joannopoulos J D and Soljacic M 2009 Nature 461772.

[37] Lepri S and Casati G 2011 Phys. Rev. Lett. 106164101.

[38] Lira H Yu Z Fan S and Lipson M 2012 Phys. Rev. Lett. 109033901.

[39] Fedotov V A Mladyonov P L Prosvirnin S L Rogacheva A V Chen Y and Zheludev N I 2006 Phys. Rev. Lett. 97167401.

[40] Menzel C Helgert C Rockstuhl C Kley E B Tünnermann A Pertsch T and Lederer F 2010 Phys. Rev. Lett. 104253902.

[41] Pfeiffer C Zhang C Ray V Gao L J and Grbic A 2014 Phys. Rev. Lett. 113023902.

[42] Fan W Wang Y Zheng R Liu D and Shi J 2015 Opt. Express 2319535.

[43] Plum E Fedotov V A and Zheludev N I 2010 J. Opt. 13024006.

[44] Verbiest T Kauranen M Van Rompaey Y and Persoons A 1996 Phys. Rev. Lett. 771456. 
Enhanced asymmetric transmission in hyperbolic epsilon-near-zero slabs

[45] Gompf B Braun J Weiss T Giessen H Dressel M and Hübner U 2011 Phys. Rev. Lett. 106185501.

[46] Ciattoni A and Spinozzi E 2013 Optik 1243623.

[47] Li L 2000 J. Opt. Soc. Am. A 17891.

[48] Menzel C Rockstuhl C and Lederer F 2010 Phys. Rev. A 82053811.

[49] Alekseyev L V Podolskiy V A and Narimanov E E 2012 Adv. Optoelectron. 2012267564.

[50] Korzeb K Gajc M and Pawlak D A 2015 Opt. Express 2325406.

[51] Cai W and Shalaev V Optical Metamaterials: Fundamentals and Applications (Springer, Dordrecht, 2010).

[52] Moharam M G Grann E B Pommet D A and Gaylord T K 1995 JOSA 121068.

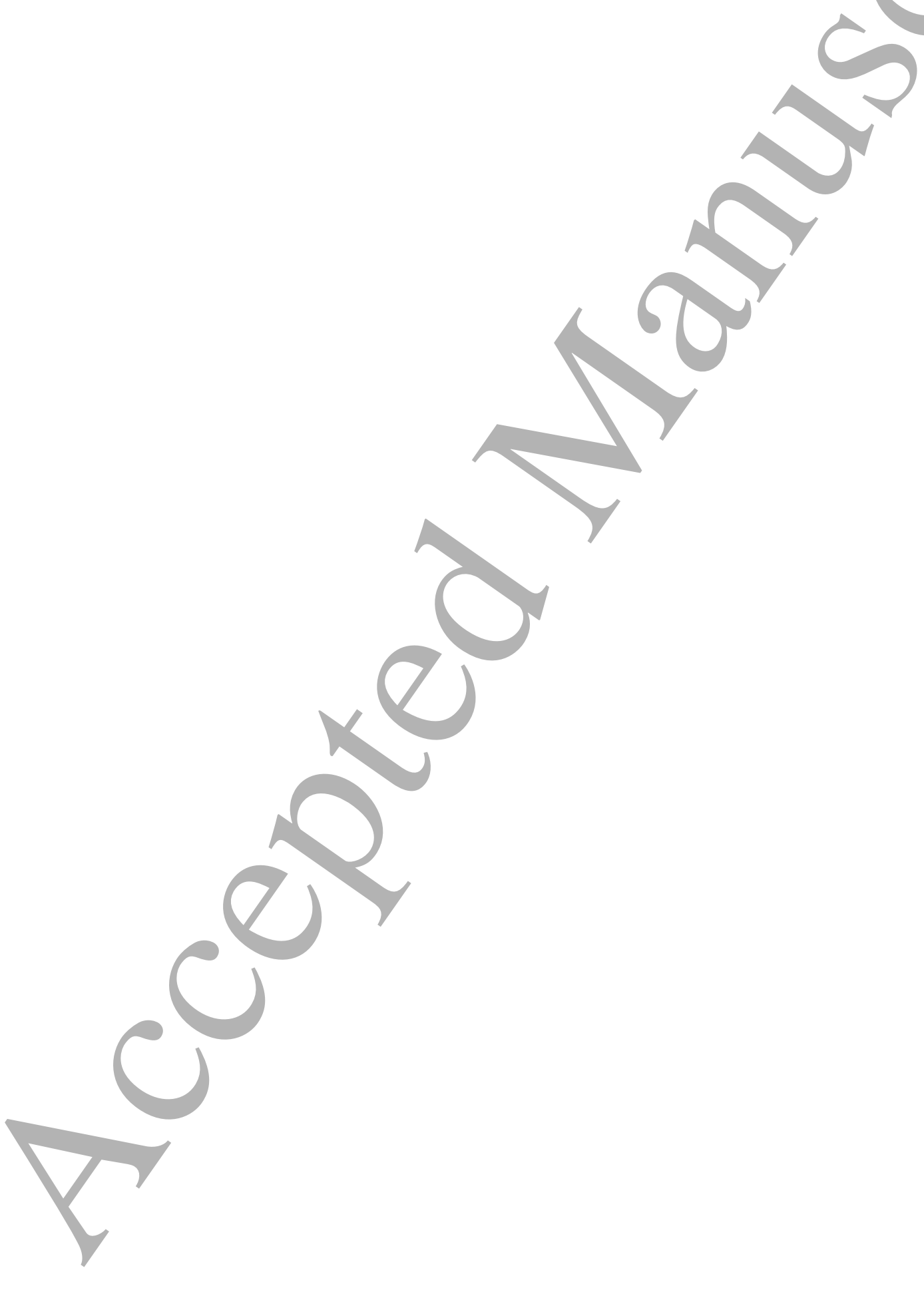

